\title{
Insect-Inspired Wall-Climbing Robots Utilizing Surface Tension Forces*
}

\author{
Kenji SUZUKI**, Shusuke NEMOTO**, Takahiro FUKUDA**, \\ Hideaki TAKANOBU ${ }^{* *}$ and Hirofumi MIURA ${ }^{* *}$ \\ ${ }^{* * K o g a k u i n ~ U n i v e r s i t y, ~}$ \\ 2665-1, Nakano-machi, Hachioji, Tokyo 192-0015, JAPAN \\ E-mail: ksuzuki@cc.kogakuin.ac.jp
}

\begin{abstract}
This paper describes insect-inspired wall-climbing robot that is capable of walking on a smooth vertical surface utilizing surface tension forces. The adhesion mechanism of the robot is inspired from the attachment system of ants, which is responsible for a thin film of secreted liquid between the adhesive organs and the surface. Two kinds of adhesive pads made of PDMS and glass were fabricated using MEMS techniques and adhesive properties were measured. Furthermore, a hexapod robot with the adhesive pads installed on its feet was developed. The robot weighs $9.5 \mathrm{~g}$ and walks in the alternating tripod gait. It successfully walked on vertical and inverted glass surfaces.
\end{abstract}

Key words: Insect-inspired Robot, Meniscus Force, Surface Tension, MEMS

\section{Introduction}

Mobile robots that are capable of climbing on vertical surfaces have many potential applications, such as surveillance, inspection, and cleaning of difficult-to-reach areas. Most of wall-climbing robots employ one of three mechanisms: vacuum suction, magnetic attraction, or grasping mechanism. Each of these mechanisms has been successful and reliable, but is limited in its range of surfaces. On the other hand, some researchers have taken a different type of attachment mechanism, found in biology, for small-scale robots. As dimensions of mechanical components decrease, adhesive forces, such as van der Waals force and capillary force, become dominant compared to gravity force. Thus, insects and other small creatures demonstrate the ability of walk on vertical walls and ceilings utilizing these adhesive forces. For instance, geckos achieve intimate contact with surfaces of varying roughness using hierarchical hairy structures, which enables "dry adhesion" based on van der Waals forces ${ }^{(1)(2)}$. Several types of gecko-inspired adhesive surfaces ${ }^{(3)}$ and wall-climbing robots have been developed ${ }^{(4)(5)}$.

This study focuses on the adhesive ability of ants utilizing "wet adhesion", which is mediated by adhesive liquid film. Ant has flexible and smooth adhesive organs called "arolium" between two claws on its feet, as shown in Fig. 1. This organ secretes adhesive fluid during walking. Recent studies reveal that rubbery deformation of the adhesive organ as well as surface tension and viscosity of the adhesive liquid contribute the adhesion and friction on the smooth surfaces ${ }^{(6)-(8)}$. The "wet adhesion" is suitable for lightweight body because the adhesive force can be obtained by applying very low normal load. In addition, secretion of liquid allows for self-cleaning of the adhesive surfaces. This study aims to develop a miniature wall-climbing robot less than 10 grams, which is much smaller than the conventional gecko-inspired robots. Therefore, the wet adhesion system was adopted.

*Received 3 Aug., 2009 (No. 09-0427) [DOI: 10.1299/jamdsm.4.383]

Copyright (C) 2010 by JSME 


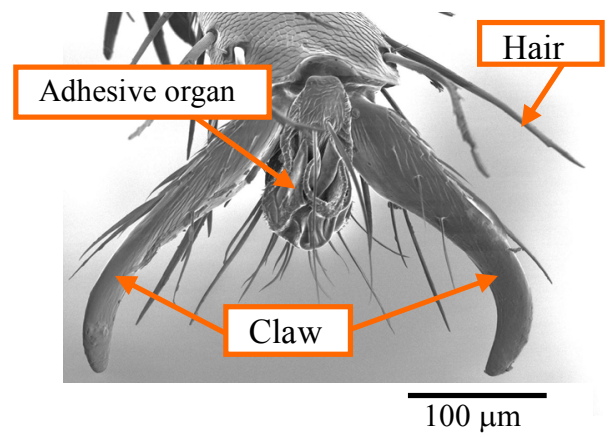

(a) SEM image of adhesive organ and claws

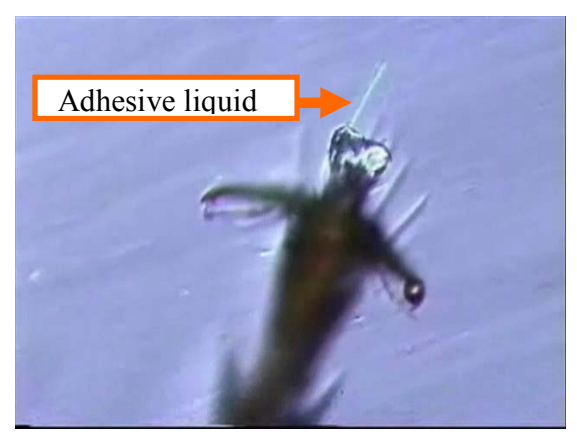

(b) Secretion of adhesive liquid

Fig. 1 Adhesive organ of ant

\section{Fabrication of adhesive pads}

Adhesive footpads inspired by ant's attachment system were fabricated using MEMS techniques. Two kinds of adhesive pads with PDMS (polydimethylsiloxian) and glass surfaces were prepared. PDMS is a kind of flexible silicone rubber and is able to adapt to any surface profile, whereas, the glass pad has a hydrophilic flat surface and generates large capillary force. Schematic drawings and a photograph of the fabricated pads are shown in Fig. 2.

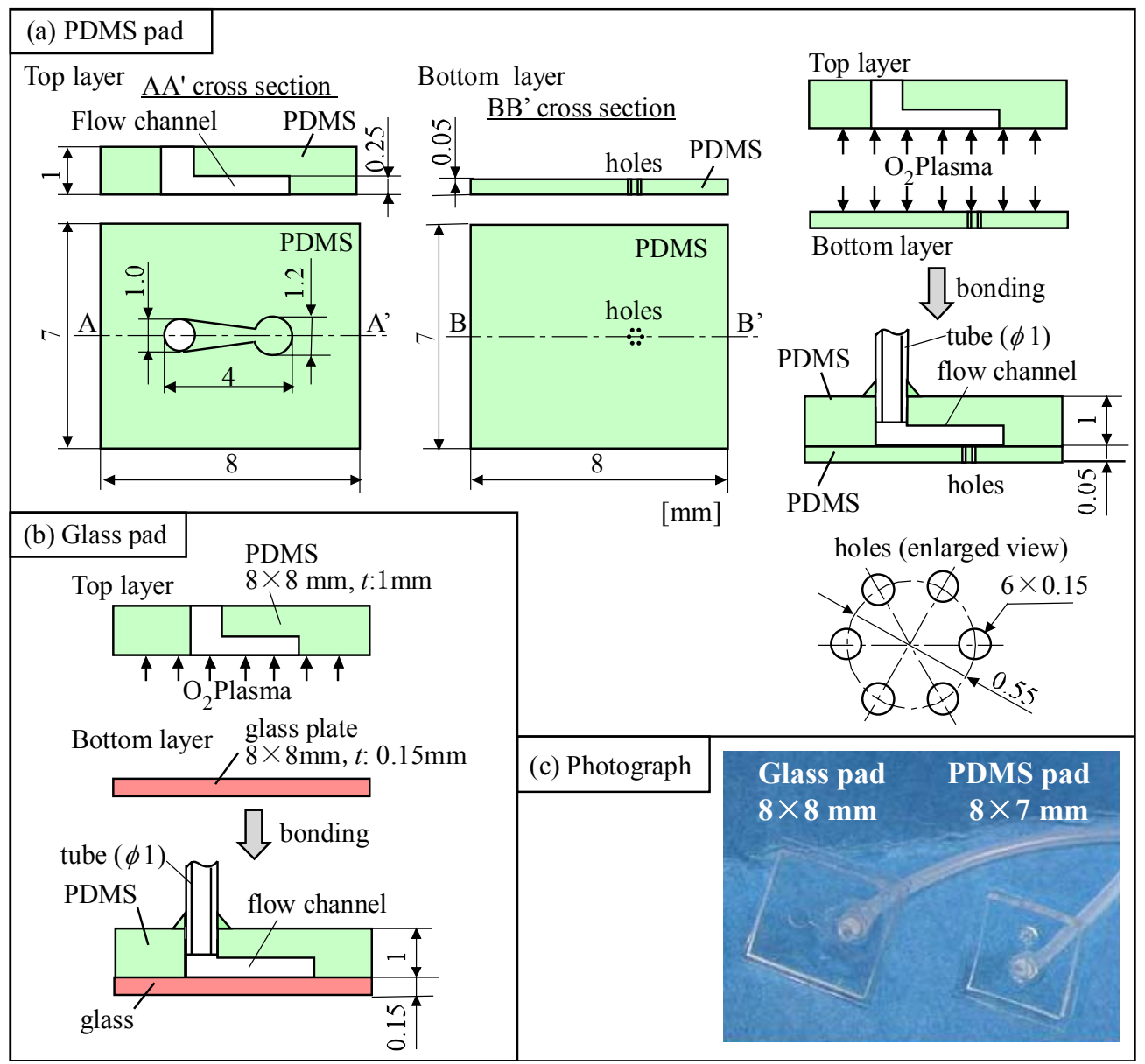

Fig. 2 Microfabricated adhesive pads 
The pad consists of a tube for supplying liquid, a flow channel, and a glass or PDMS adhesive layer. In the adhesive layer of the PDMS pad, six holes of $150 \mu \mathrm{m}$ in diameter are fabricated for liquid secretion. Sizes of the glass pad and the PDMS pad are $8 \times 8 \mathrm{~mm}$ and $8 \times 7 \mathrm{~mm}$, respectively. The adhesive bottom layer and the flow channel in the top layer were fabricated separately using molds of photoresist (SU8) and bonded with $\mathrm{O}_{2}$ plasma treatment.

\section{Measurement of adhesive forces}

Normal and tangential forces of the adhesive pads on a flat glass surface were measured. In this experiment, deionized water is used for the adhesive liquid and supplied externally using a micropipette. The experimental setup for measuring normal and tangential forces is shown in Fig. 3. The adhesive pad was first loaded on the glass wafer and then pulled-off for measuring normal force, or slid on the wafer for measuring tangential force using a 3-axis positioning stage. Deformation of the double cantilever spring, shown in Fig. 4, was detected using a laser displacement sensor and the adhesive forces were calculated. Figure 5 shows the typical data of the normal force. The normal load is obtained as a negative value of the adhesive force. The normal adhesive force corresponds to the maximum value of the pull-off force.

The experimental results for the PDMS pads are shown in Fig. 6. Normal adhesive force shows strong dependence on normal load and does not depend on the volume of water. The tangential force of the PDMS pad is relatively large and does not depend on the volume of water. These results suggest that the adhesive force of the PDMS pad is primarily responsible for van der Waals force between the pad and the glass surface. Large normal load deforms the PDMS pad and increases the contact area, which results in increase in normal adhesive force and tangential friction.

Experimental results for the glass pad are shown in Fig. 7. Normal adhesive force shows dependence on the volume of water and reaches maximum at $0.3 \mu \mathrm{l}$. In contrast, the tangential force remains low regardless of the volume of water. The normal adhesive force is much larger than the tangential force. These results suggest that adhesion of the glass pad is responsible for meniscus force.

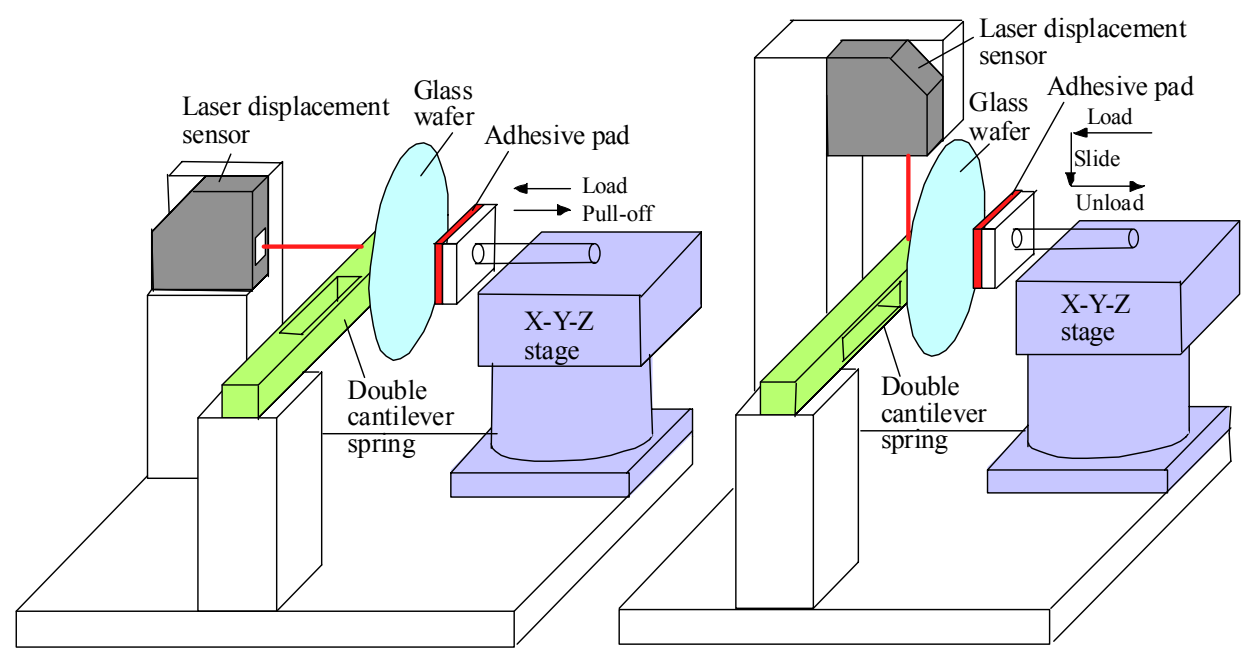

(a) Measurement of normal force

(b) Measurement of tangential force

Fig. 3 Experimental setups for measuring adhesive forces 


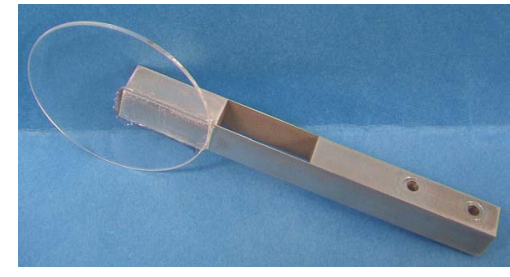

Fig. 4 Double cantilever spring

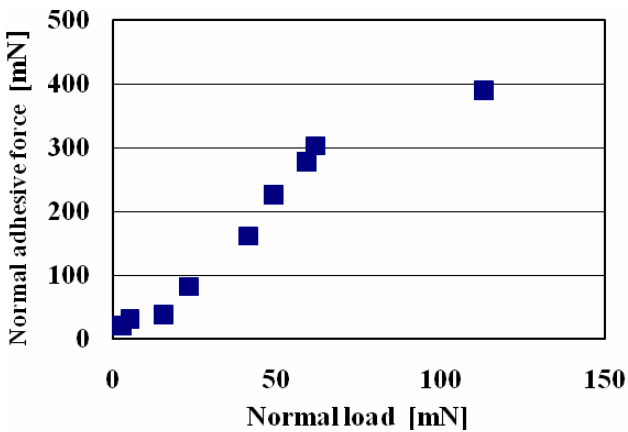

(a) Normal adhesive force as a function of normal load

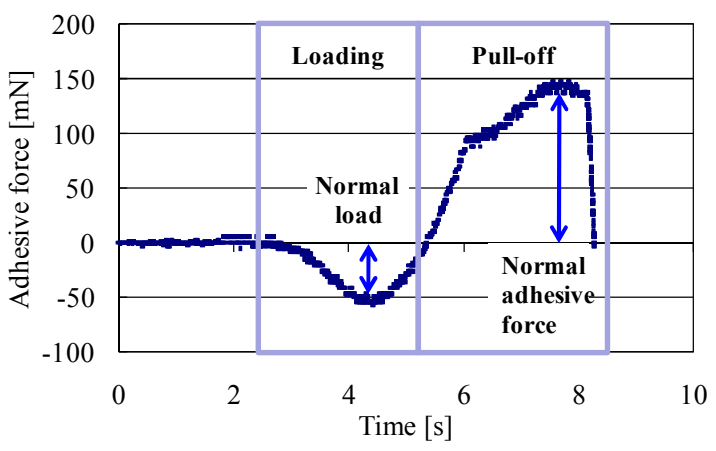

Fig. 5 Obtained data of normal adhesive force

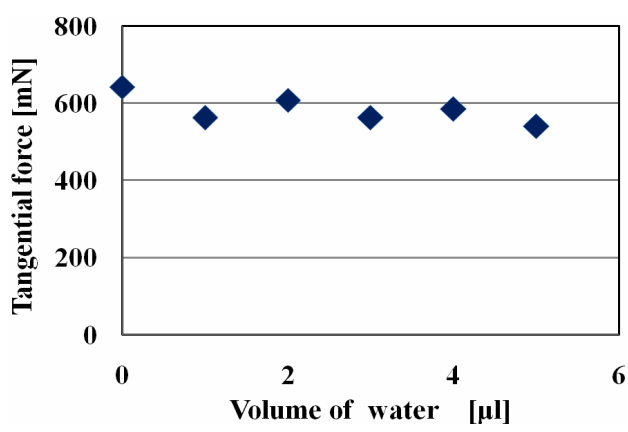

(b) Tangential force as a function of volume of water

Fig. 6 Adhesive forces of PDMS pad

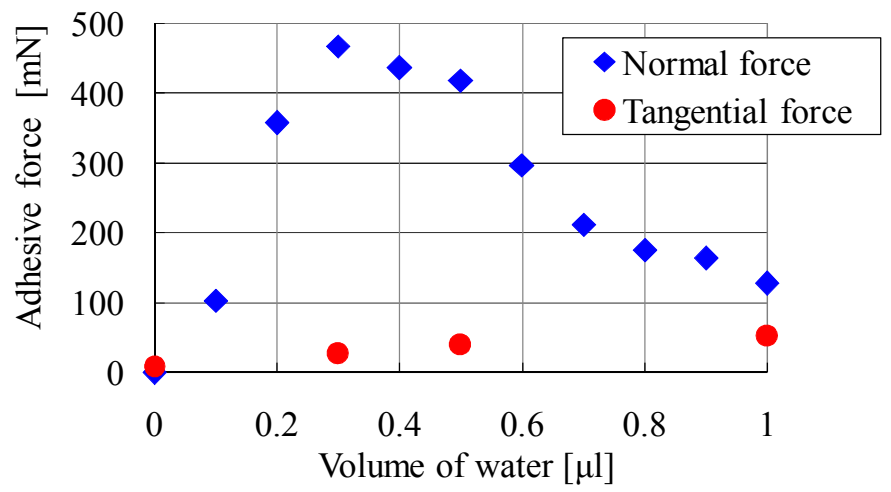

Fig. 7 Adhesive forces of glass pad

Normal and tangential meniscus forces acting between two parallel surfaces are schematically illustrated in Fig. 8. When the surface is hydrophilic, the liquid droplet forms concave meniscus, which generates negative Laplace pressure:

$$
p=\gamma\left(-\frac{1}{r_{1}}+\frac{1}{r_{2}}\right)<0
$$

where $\gamma$ is surface tension of the liquid, $r_{1}$ and $r_{2}$ are radii of curvatures of the meniscus. The normal meniscus force consists of two components: attractive force caused by negative Laplace pressure and vertical component of surface tension force acting on the gas-liquid-solid contact line: 


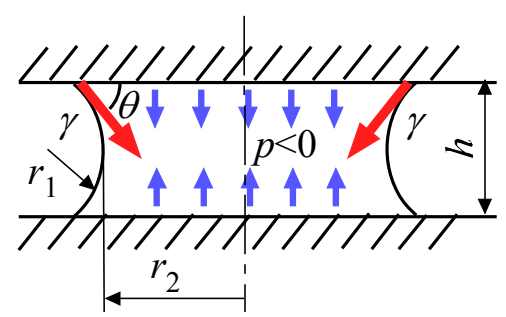

(a) Normal force

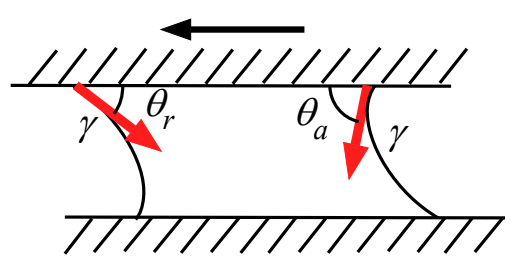

(b) Tangential force

Fig.8 Meniscus forces in normal and tangential directions

$$
F_{n}=\pi r_{2}^{2} \gamma\left(\frac{1}{r_{1}}-\frac{1}{r_{2}}\right)+2 \pi r_{2} \gamma \sin \theta
$$

where $\theta$ is contact angle of the liquid. If $r_{1} \ll r_{2}$, the contribution of Laplace pressure is dominant.

$$
\left.F_{n} \approx \pi \gamma \frac{r_{2}^{2}}{r_{1}} \quad \text { (if } r_{1} \ll r_{2}\right)
$$

Relation between the radii $r_{1}, r_{2}$ and the volume of water $V$ is given by

$$
V=\pi r_{2}^{2} h=2 \pi r_{2}^{2} r_{1} \cos \theta
$$

where $h$ is the thickness of the liquid film. As the volume of water $V$ increases, normal adhesive force $F_{n}$ increases because of increase in $r_{2}$. However, $r_{2}$ is limited by the size of the pad. After the liquid spreads to the whole area of the pad, the thickness $h$ increases and $F_{n}$ decreases. Thus, there is a peak of the normal adhesive force.

The tangential meniscus force $F_{t}$ is given by integral of horizontal components of surface tension force acting on the gas-liquid-solid contact line $l$.

$$
F_{t}=\oint_{l} \gamma \cos \theta d l \approx 2 \gamma r_{2}\left(\cos \theta_{r}-\cos \theta_{a}\right)
$$

where $\theta_{a}$ and $\theta_{r}$ are advancing and receding contact angles, respectively. The tangential force is caused by the contact angle hysteresis $\left(\theta_{a}-\theta_{r}\right)$. If $r_{1} \ll r_{2}$, the normal force $F_{n}$ is much larger than tangential force $F_{t}$.
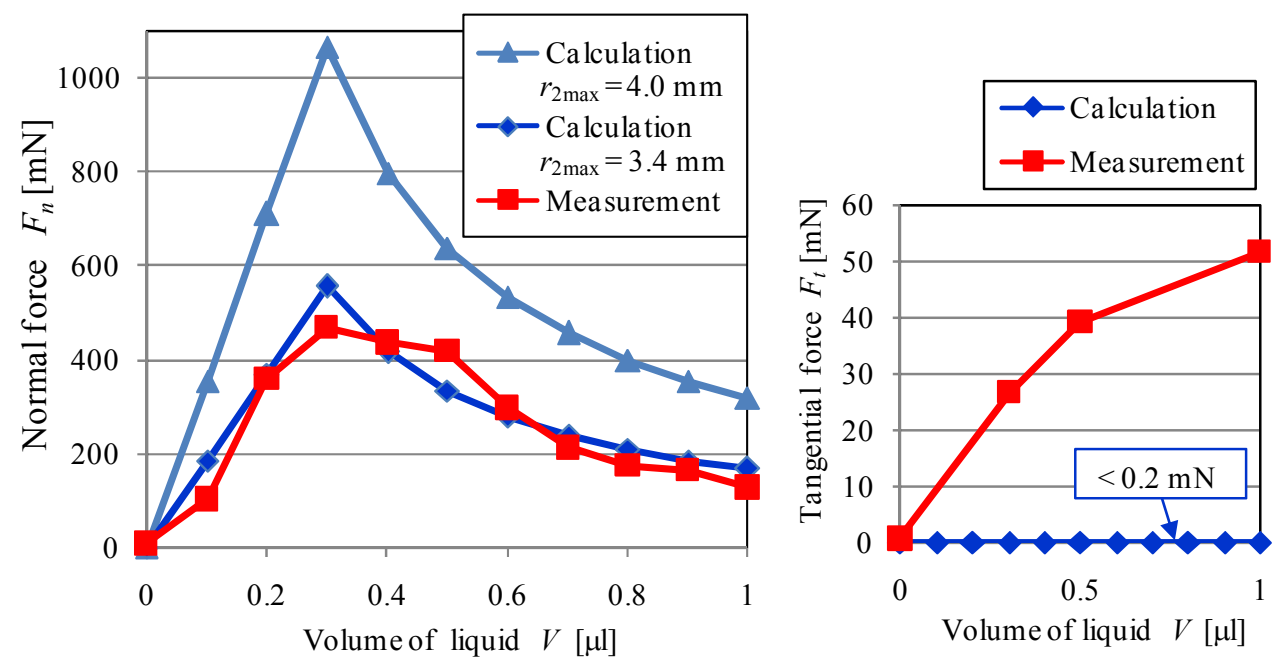

Fig.9 Comparison between measurements and numerical calculations 
Figure 9 shows the comparison between measurements and numerical calculations of the adhesive forces. It is assumed that $r_{2}$ reaches a maximum value $(4 \mathrm{~mm}$ or $3.4 \mathrm{~mm})$ at $V=0.3 \mu \mathrm{l}$ and remains constant in the range of $V>0.3 \mu \mathrm{l}$. The measured normal forces are in good agreement with the calculated meniscus force under the condition of $r_{2 \max }=3.4$ $\mathrm{mm}$, although the actual radius $r_{2 \max }$ is approximately $4 \mathrm{~mm}$ (a half width of the glass pad). This difference is due to the roughness of the glass surfaces. Measured tangential forces are much larger than calculated one. These results suggest that dry friction between the surface asperities primarily contributes to the tangential adhesive force.

\section{Wall-Climbing Robot}

Taking advantage of the adhesive properties of glass and PDMS pads, a hexapod wall-climbing robot was designed. Six PDMS pads were attached to each foot of the robot to generate tangential friction. In addition, a glass pad was installed on the abdomen of the robot for normal adhesion. Deionized water of $0.3 \mu \mathrm{l}$ was supplied on the glass pad using a micropipette to generate a maximum adhesive force. The glass pad remains in contact with the surface and slides with low tangential force during walking. The mechanism for generating the leg motion is shown in Fig. 10. The tip of the leg moves along a triangular orbit so that the PDMS pads can be attached perpendicular to the wall and detached at a slant angle. The robot walks in the "alternating tripod gait" shown in Fig. 11, which is one

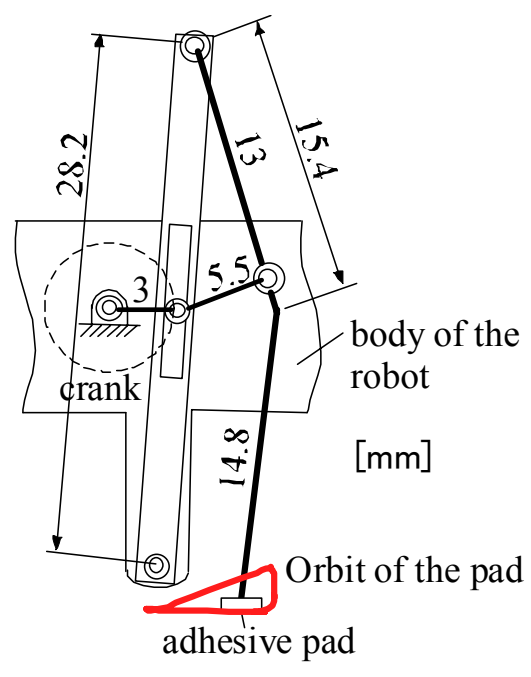

(a) Mechanism of a leg

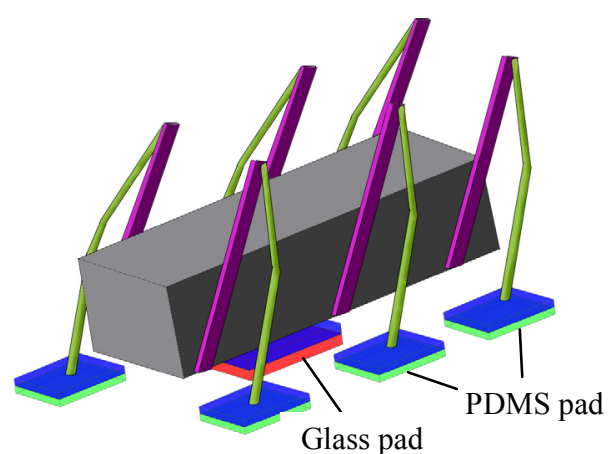

(b) Arrangement of the glass and PDMS pads

Fig. 10 Mechanism for the leg motion.

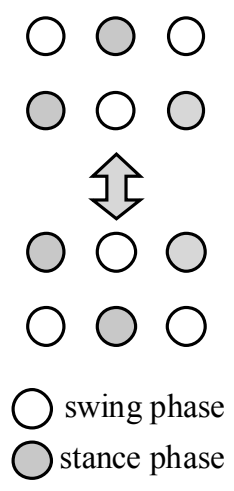

Fig. 11 Alternating tripod gait

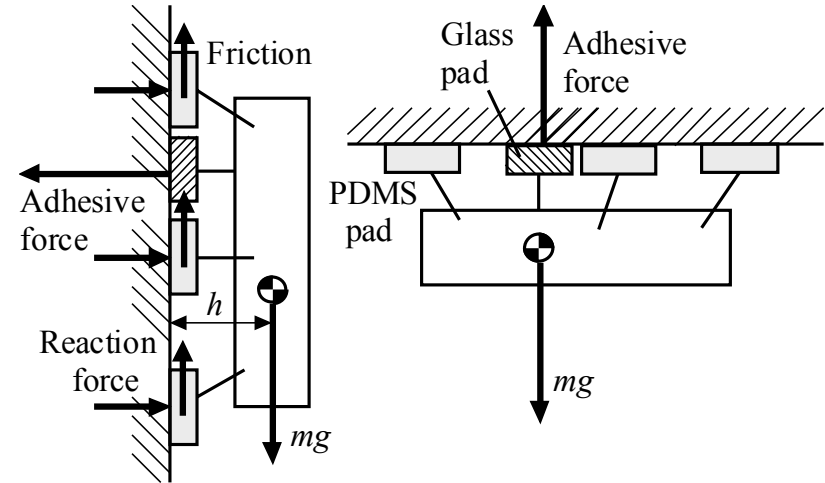

Fig. 12 Force and moment balance of the robot 


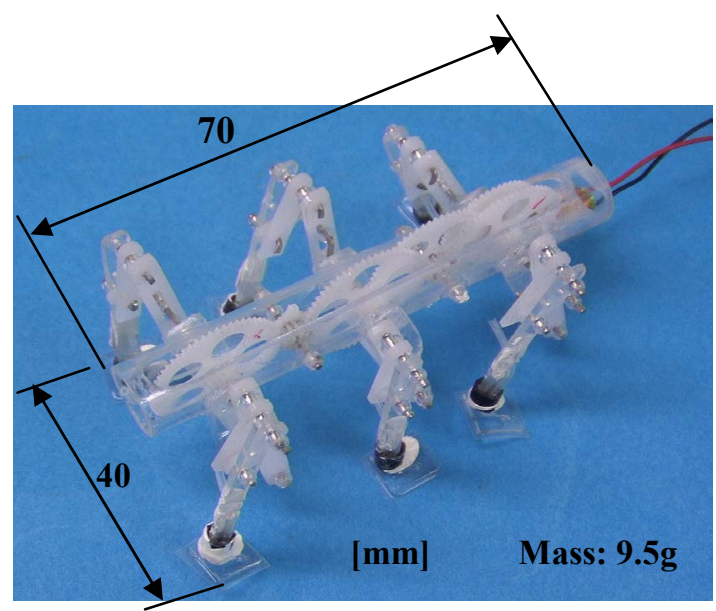

(a) top view

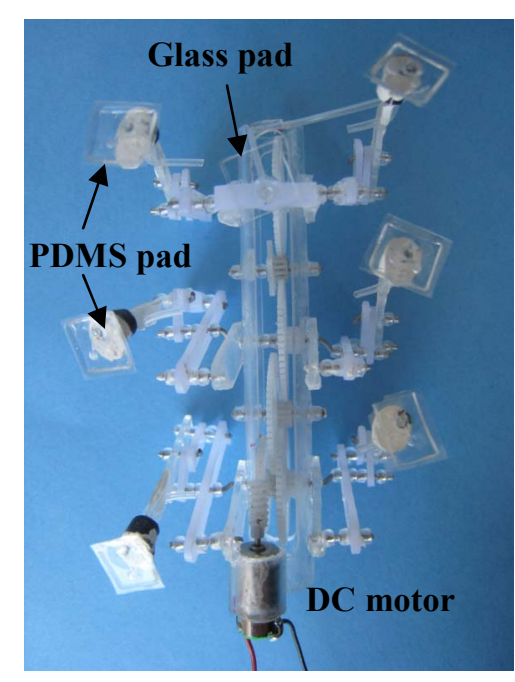

(b) bottom view

Fig. 13 Photographs of the robot

of typical gaits of insects. Force and moment balance of the robot walking on a vertical wall and a ceiling is shown in Fig. 12. On the vertical wall, the weight of the robot is supported by tangential friction of the PDMS pads. The glass pad is attached on a front part of the body to support the moment caused by the gravity that pulls the body away from the surface. The total mass of the robot is designed as $9.5 \mathrm{~g}$ including a DC motor and a gear box so that three PDMS pads can sufficiently support the weight during tripod walking. When the robot walks on the ceiling, the glass pad is placed in the center part of the body to support the weight. Tangential forces of the PDMS pads are used to obtain the propulsive force. The photographs of the robot are shown in Fig. 13. The length of the robot is $70 \mathrm{~mm}$.

The experiments for the locomotion of the robot on vertical and inverted glass surfaces were carried out, as shown in Fig. 14. The robot successfully walked on both the vertical wall and the ceiling in the alternating tripod gait. A period of the leg motion including swing and stance phases was approximately 6 seconds. The walking speed was approximately 2.8 $\mathrm{mm} / \mathrm{s}$ on the horizontal surface and ceiling. It was slightly slower on the vertical surface because the PDMS pads were apt to slip just before the detachment of the pad. The normal load and the friction of the PDMS pads can be adjusted by changing the clearance between the glass pad and the surface. When the clearance was $1.2 \mathrm{~mm}$, the slip of the pad was minimized.

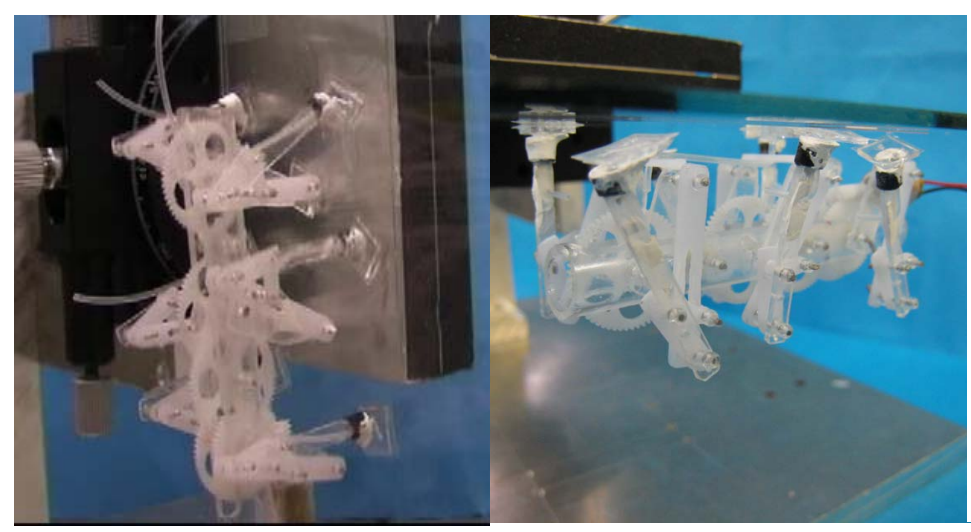

Fig. 14 Photographs of the robot walking on a vertical wall and a ceiling 


\section{Conclusions}

Glass and PDMS adhesive pads inspired by ant's attachment devices were fabricated with MEMS techniques. Adhesive properties of the pads were investigated experimentally. The glass pad shows large normal adhesive force and small tangential friction under wet conditions, whereas, the PDMS pad exhibits large friction under dry condition. A hexapod wall-climbing robot using two kinds of adhesive pads was developed. It successfully walked on vertical and inverted glass surfaces in the alternating tripod gait.

\section{Acknowledgement}

This work was supported by the Biomedical Engineering Research Center (BERC) at Kogakuin University. Matching funding was received from the Ministry of Education, Culture, Sports, Science and Technology (MEXT), 2008-2012. This research was also partially supported by the MEXT, Grant-in-Aid for Scientific Research (B), 19360121, 2007-2009.

\section{References}

(1) K. Autumn, et al., "Adhesive force of a single gecko foot-hair," NATURE, Vol. 405, 2000, pp.681-685,

(2) Y. Tian, et al., "Adhesion and friction in gecko toe attachment and detachment," Proc. the National Academy of Sciences, Vol. 103, No. 51, 2006, pp.19320-19325.

(3) M. Sitti and R. S. Fearing, "Synthetic Gecko Foot-hair Micro/Nano-Structures as Dry Adhesives," Journal of Adhesion Science and Technology, Vol. 17, No. 8, 2003, pp. 1055-1074.

(4) S. Kim, M. Spenko, S. Trujillo, B. Heyneman, D. Santos, and M. R. Cutkosky, "Vertical Surface Climbing With Directional Adhesion," IEEE Transactions on Robotics, Vol.24,No.1, 2008, pp.65-74

(5) M. P. Murphy and M. Sitti, "Waalbot: An Agile Small-Scale Wall-Climbing Robot Utilizing Dry Elastomer Adhesives," IEEE Transactions on Mechatronics, Vol. 12, No.3, 2007, pp.330-338.

(6) S. N. Gorb, "Smooth attachment Devices in Insects: Functional Morphology and Biomechanics," in Advances in Insect Physiology, Vol. 34, edited by J. Casas, and S. J. Simpson, Academic Press, 2008, pp.81-115.

(7) W. Federle, et al., "Biomechanics of ant adhesive pads: frictional forces are rate- and temperature- dependent," The Journal of Experimental Biology, Vol. 207, 2004, pp. 67-74.

(8) W. Federle, M. Riehle, A. S. G. Curtis, and R. J. Full, "An Integrative Study of Insect Adhesion: Mechanics and Wet Adhesion of Pretarsal Pads in Ants," Integrative and Comparative Biology, Vol. 42, 2002, pp.1100-1106. 